\title{
Detecting the neutral top-pion at future muon colliders
}

\author{
Chongxing Yue ${ }^{a}$, Lanjun $\mathrm{Liu}^{b}$, Dongqi Yu ${ }^{a}$ \\ a: Department of Physics, Liaoning Normal University, Dalian 116029. P.R.China * \\ b:College of Physics and Information Engineering, \\ Henan Normal University, Xinxiang 453002. P.R.China
}

June 20, 2021

\begin{abstract}
The signals of the neutral top-pion $\pi_{t}^{0}$ at the first muon collider (FMC) are discussed by calculating its contributions to the processes $\mu^{+} \mu^{-} \longrightarrow b \bar{b}$ and $\mu^{+} \mu^{-} \longrightarrow \bar{t} c$. We find that the contributions to the process $\mu^{+} \mu^{-} \longrightarrow b \bar{b}$ are very small and the ratio of signal over square root of the background $(S / \sqrt{B})$ is smaller than 0.2. However, in most of the parameter space, the $\pi_{t}^{0}$ can generate 36 and up to 649 observable $\bar{t} c$ events at the FMC. The signals of the neutral top-pion $\pi_{t}^{0}$ may be detected via the process $\mu^{+} \mu^{-} \longrightarrow \bar{t} c$ at the FMC.
\end{abstract}

PACS number(s):12.60Nz,14.80.Mz,12.15.Lk,14.65.Ha

*E-mail:cxyue@lnnu.edu.cn 


\section{Introduction}

A muon collider is an excellent tool to study the properties of a heavy scalar or pseudoscalar $[1,2]$. It is thought that the first muon collider (FMC) can achieve the same integrated luminosity and energy as a high energy $e^{+} e^{-}$collider. The FMC can explore all the same physics that is accessible at an $e^{+} e^{-}$collider of the same energy. Furthermore, it has been shown that a large number of Higgs bosons [1] or new particles, such as technihadrons and technipions [3,4], can be produced through an s-channel resonance process and the flavor changing scalar (FCS) couplings [5] can be tested at the FMC. Thus, the FMC makes possible precise measurements of the total widths and masses of the various neutral particles, which will open a window towards the new physics.

Until a Higgs boson with large coupling to a gauge boson pair is discovered, the possibility of the electroweak symmetry broken by new strong interactions still exists [6]. The most commonly studied class of theories is technicolor (TC) [7], which dynamically breakes the electroweak symmetry. Although TC models have many theoretical problems as well as conflict with data and broad classes of these models have been ruled out, there are still viable models worthy of investigation in light of the capabilities of the current collider experiments. Topcolor-assisted technicolor (TC2) models [8] are such type of examples.

The common feature of all TC2 models is that topcolor interactions generate the main part of large top quark mass and only make small contributions to electroweak symmetry breaking (EWSB). In order that topcolor interactions are natural, i.e. without introducing large isospin violation, it is necessary that EWSB is still mainly generated by TC interactions. For TC2 models, ETC interactions are still needed to generate the masses of light quarks and contribute a few $G e V$ to top quark mass, i.e. $\varepsilon m_{t}$ with $\varepsilon \ll 1$ [8]. Thus, the presence of a number of pseudo Goldstone bosons (PGB's), including the technipions in TC sector and three top-pions $\left(\pi_{t}^{ \pm, 0}\right)$ in topcolor sector, in the low-energy spectrum is an inevitable feature of these models. These new particles are most directly related to EWSB. Thus, studying the possible signatures of these new particles at present and future high energy experiments would provide crucial information for EWSB and 
fermion flavor physics as well.

The production and decay of the technipions and top-pions have been extensively studied in several instances $[9,10]$. Combining resonance and non-resonance contributions, the signals of technipions are recently studied at lepton colliders and hadron colliders [11]. Ref.[12] has discussed the single production of the neutral top-pion $\pi_{t}^{0}$ at hadron colliders and high energy $e^{+} e^{-}$colliders (LCs). The observability of the neutral top-pion $\pi_{t}^{0}$ and the charged top-pions $\pi_{t}^{ \pm}$has been studied via considering the single top production at hadron colliders and linear colliders $[13,14]$.

The effects of the top-pion on physical observables are governed by its mass $m_{\pi_{t}}$, while the large couplings of top-pions to quarks and to gauge bosons are to large degree modelindependent [15]. Thus, if the neutral top-pion $\pi_{t}^{0}$ is discovered at the LHC or other future collider experiments, it is need to consider its resonance production at the FMC, which can make possible precise measurements of the couplings and mass of the neutral top-pion $\pi_{t}^{0}$. In this paper, we consider the s-channel resonance production of the neutral top-pion $\pi_{t}^{0}$ at the FMC with the centre-of-mass energy $\sqrt{s}=200 G e V-500 G e V$ and explore the potential of the FMC for detecting this new particle.

This paper is organized as follows: in section 2, we give the possible couplings of the neutral top-pion $\pi_{t}^{0}$ to the ordinary particles. The resonance production cross section $\sigma(b \bar{b})$ of the process $\mu^{+} \mu^{-} \longrightarrow \pi_{t}^{0} \longrightarrow b \bar{b}$ and the ratio of signal over square root of the background $(S / \sqrt{B})$ are calculated in section 3. For TC2 models, the topcolor interactions are non-universal, the neutral top-pion $\pi_{t}^{0}$ has large FCS coupling $\pi_{t}^{0} \bar{t} c$, the possibility of detecting $\pi_{t}^{0}$ via the process $\mu^{+} \mu^{-} \longrightarrow \pi_{t}^{0} \longrightarrow \bar{t} c$ is studied in section 3. Our conclusions are given in section 4 .

\section{The couplings of the neutral top-pion $\pi_{t}^{0}$ to ordinary particles}

For TC2 models [8], TC interactions play a main role in breaking the electroweak symmetry. Topcolor interactions make small contributions to EWSB, and give rise to the main part of the top quark mass, $(1-\varepsilon) m_{t}$, with the parameter $\varepsilon \ll 1$. Thus, there is 
the following relation:

$$
\nu_{\pi}^{2}+F_{t}^{2}=\nu_{W}^{2}
$$

where $\nu_{\pi}$ represents the contributions of TC interactions to EWSB, $\nu_{W}=\nu / \sqrt{2}=$ $174 \mathrm{GeV} . F_{t}=50 \mathrm{GeV}$ is the physical top-pion decay constant, which can be estimated from the Pagels-Stokar formula. This means that the masses of gauge bosons $\mathrm{W}$ and $\mathrm{Z}$ are given by absorbing the linear combination of the top-pions and technipions. The orthogonal combination of the top-pions and technipions remains unabsorbed and physical. However, the absorbed Goldstone linear combination is mostly the technipions while the physical linear combination is mostly the top-pions, which are usually called physical top-pions $\left(\pi_{t}^{ \pm}, \pi_{t}^{0}\right)$. The existence of the physical top-pions in the low-energy spectrum can be seen as characteristics of topcolor scenario, regardless of the dynamics responsible for EWSB and other quark masses [15].

For TC2 models, the underlying interactions, topcolor interactions, are non-universal and therefore do not posses a GIM mechanism. The non-universal gauge interactions result in the new $\mathrm{FC}$ coupling vertices when one writes the interactions in the quark mass eigen-basis. Thus, the top-pions have large Yukawa couplings to the third generation quarks and can induce the new FCS couplings. The couplings of the neutral top-pion $\pi_{t}^{0}$ to the third generation quarks including the $t-c$ transition can be written as $[8,14]$ :

$$
\frac{m_{t}}{\sqrt{2} F_{t}} \frac{\sqrt{\nu_{W}^{2}-F_{t}^{2}}}{\nu_{W}}\left[K_{U R}^{t t} K_{U L}^{t t} \bar{t}_{L} t_{R} \pi_{t}^{0}+\frac{m_{b}-m_{b}^{\prime}}{m_{t}} \bar{b}_{L} b_{R} \pi_{t}^{0}+K_{U R}^{t c} K_{U L}^{t t} \overline{t_{L}} c_{R} \pi_{t}^{0}+\text { h.c. }\right],
$$

where $m_{b}^{\prime}$ is the ETC generated part of the bottom-quark mass. Similar to Ref.[10], we take $m_{b}^{\prime}=0.1 \varepsilon m_{t} . \quad K_{U L}^{t t}$ is the matrix element of the unitary matrix $K_{U L}$ which the CKM matrix can be derived as $V=K_{U L}^{-1} K_{D L}$ and $K_{U R}^{i j}$ are the matrix elements of the right-handed rotation matrix $K_{U R}$. Their values can be written as [14] :

$$
K_{U L}^{t t}=1, \quad K_{U R}^{t t}=1-\varepsilon, \quad K_{U R}^{t c} \leq \sqrt{2 \varepsilon-\varepsilon^{2}}
$$

In the following calculation, we will take $K_{U R}^{t c}=\sqrt{2 \varepsilon-\varepsilon^{2}}$ and take the parameter $\varepsilon$ as a free parameter. 
The couplings of the neutral top-pion $\pi_{t}^{0}$ to the first, second generation fermions and the third generation leptons can be written as :

$$
\frac{m_{f}}{\sqrt{2} F_{t}} \frac{F_{t}}{\nu_{W}} \bar{f} \gamma^{5} f \pi_{t}^{0}=\frac{m_{f}}{\nu} \bar{f} \gamma^{5} f \pi_{t}^{0} .
$$

The neutral top-pion $\pi_{t}^{0}$, as an isospin-triplet, can couple to a pair of gauge bosons through the top quark trangle loop in an isospin violating way similar to the couplings of QCD pion $\pi^{0}$ to a pair of gauge bosons. The relevant formula of these couplings has been given in Ref.[10].

Ref.[8] has estimated the mass of the top-pion in the fermion loop approximation and given $180 \mathrm{GeV} \leq m_{\pi_{t}} \leq 240 \mathrm{GeV}$ for $m_{t}=175 \mathrm{GeV}$ and $0.03 \leq \varepsilon \leq 0.1$. The limits on the mass of the top-pion may be obtained via studying its effects on various experimental observables. For example, Ref.[16] has shown that the process $b \longrightarrow s \gamma, B-\bar{B}$ mixing and $D-\bar{D}$ mixing demand that the top-pions are likely to be light, with masses of the order of a few hundred $G e V$. Since the negative top-pion corrections to the $Z \longrightarrow b \bar{b}$ branching ratio $R_{b}$ become smaller when the top-pion is heavier, the LEP-SLD data of $R_{b}$ give rise to a certain lower bound on the top-pion mass [15]. It was shown that the top-pion mass should not be lighter than the order of $1 \mathrm{TeV}$ to make TC2 models consistent with the LEP-SLD data [17]. We restudied the problem in Ref.[18] and find that the top-pion mass $m_{\pi_{t}}$ is allowed to be in the range of a few hundred $G e V$ depending on the models. Thus, the value of the top-pion mass $m_{\pi_{t}}$ remains subject to large uncertainty [7]. Furthermore, Refs. $[13,14]$ have shown that the top-pion mass $m_{\pi_{t}}$ can be explored up to $300-350 \mathrm{GeV}$ via the processes $p \bar{p} \longrightarrow \pi_{t}^{0} \longrightarrow \bar{t} c$ and $p \bar{p} \longrightarrow \pi_{t}^{ \pm} x$ at the Tevatron and LHC. Thus, we will take $m_{\pi_{t}}$ as a free parameter and assume it to vary in the range of $200 \mathrm{GeV}-400 \mathrm{GeV}$ in this paper. In this case, the possible decay modes of $\pi_{t}^{0}$ are $b \bar{b}, \bar{t} c, \bar{f} f(f$ is the first or second generation fermions or the third generation leptons), $t \bar{t}$ (if kinematically allowed), $g g, \gamma \gamma$, and $Z \gamma$. The total decay width of $\pi_{t}^{0}$ can be written as:

$$
\begin{aligned}
\Gamma_{\pi_{t}} & =\Gamma\left(\pi_{t}^{0} \longrightarrow b \bar{b}\right)+\Gamma\left(\pi_{t}^{0} \longrightarrow \bar{t} c\right)+\Gamma\left(\pi_{t}^{0} \longrightarrow f \bar{f}\right)+\Gamma\left(\pi_{t}^{0} \longrightarrow g g\right) \\
& +\Gamma\left(\pi_{t}^{0} \longrightarrow \gamma \gamma\right)+\Gamma\left(\pi_{t}^{0} \longrightarrow Z \gamma\right)+\Gamma\left(\pi_{t}^{0} \longrightarrow t \bar{t}\right)\left(m_{\pi_{t}} \geq 2 m_{t}\right) .
\end{aligned}
$$




\section{The signals of the neutral top-pion $\pi_{t}^{0}$ at the FMC}

From above discussions, we can see that the neutral top-pion $\pi_{t}^{0}$ can be produced at the FMC, operating at a centre-of-mass energy $\sqrt{s}$ of up to $500 \mathrm{GeV}$. In spite of the fact that the coupling $\pi_{t}^{0} \mu^{+} \mu^{-}$, being proportional to $m_{\mu} / \nu$, is very small, if the FMC run on the $\pi_{t}^{0}$ resonance $\left(\sqrt{s}=m_{\pi_{t}}\right)$, the $\pi_{t}^{0}$ may be produced at an appreciable rate. For $m_{\pi_{t}} \leq 2 m_{t}$, the main decay modes of $\pi_{t}^{0}$ are $\bar{t} c, b \bar{b}$ and $g g$. So, in this section, we will consider the possibility of detecting $\pi_{t}^{0}$ via the processes $\mu^{+} \mu^{-} \longrightarrow \pi_{t}^{0} \longrightarrow b \bar{b}$ and $\mu^{+} \mu^{-} \longrightarrow \pi_{t}^{0} \longrightarrow \bar{t} c$ at the FMC.

\section{The neutral top-pion $\pi_{t}^{0}$ and the process $\mu^{+} \mu^{-} \longrightarrow b \bar{b}$}

Convoluted with the collider energy distribution, the s-channel resonance cross section for production of a final state $x$ generated by the neutral top-pion $\pi_{t}^{0}$ at the FMC is given by [1]:

$$
\sigma(x) \approx \frac{4 \pi}{m_{\pi_{t}}^{2}} \frac{B_{r}\left(\pi_{t}^{0} \longrightarrow \mu^{+} \mu^{-}\right) B_{r}\left(\pi_{t}^{0} \longrightarrow x\right)}{\left[1+\frac{8}{\pi}\left(\frac{\sigma_{\sqrt{s}}}{\Gamma_{\pi_{t}}}\right)^{2}\right]^{1 / 2}} .
$$

The Gaussian spread in the beam energy $\sqrt{s}$ is given by $\sigma_{\sqrt{s}}=\frac{R}{\sqrt{2}} \sqrt{s}$. The energy resolution $R$ of each beam is expected to be in the range of $0.003 \%-0.05 \%$ and we will take $R=0.03 \%$.

In Fig.1, we plot the resonance production section $\sigma(b \bar{b})$ versus the top-pion mass $m_{\pi_{t}}$ for $\sqrt{s}=m_{\pi_{t}}$ and three values of the parameter $\varepsilon$. We can see from Fig.1 that $\sigma(b \bar{b})$ decreases with the top-pion mass $m_{\pi_{t}}$ and the parameter $\varepsilon$ increasing. The value of $\sigma(b \bar{b})$ is in the range of $2.1 \mathrm{fb}-0.01 \mathrm{fb}$ for $0.02 \leq \varepsilon \leq 0.08$ and $200 \mathrm{GeV} \leq m_{\pi_{t}} \leq 350 \mathrm{GeV}$. Thus, there may be $1-42 b \bar{b}$ events to be produced at the FMC with $\sqrt{s}=200 \mathrm{GeV}-500 \mathrm{GeV}$ and a yearly integrated luminosity of $L=20 \mathrm{fb}^{-1}$. To see whether the neutral top-pion $\pi_{t}^{0}$ can be detected via the process $\mu^{+} \mu^{-} \longrightarrow b \bar{b}$ at the FMC, it is need to further calculate the ratio of signal over square root of the background $\frac{S}{\sqrt{B}}$.

To solve the phenomenological difficulties of the traditional TC models [7], TC2 models [8] were proposed by combining technicolor interactions with topcolor interactions for the third generation quarks. TC2 models predict a number of technipions in the TC sector. Refs. $[3,4]$ have pointed out that the color-singlet technipion $P^{0}$ can be significantly 
produced in the s-channel. Thus, the backgrounds of the process $\mu^{+} \mu^{-} \longrightarrow \pi_{t}^{0} \longrightarrow b \bar{b}$ mainly come from the exchange of the gauge bosons $\gamma, Z$ and of the color-singlet technipion $P^{0}$ in the s-channel. We calculate the value of $\frac{S}{\sqrt{B}}$ and find that it is smaller than 0.2 in all of the parameter space. So the neutral top-pion $\pi_{t}^{0}$ can not be detected via the process $\mu^{+} \mu^{-} \longrightarrow b \bar{b}$ at the FMC.

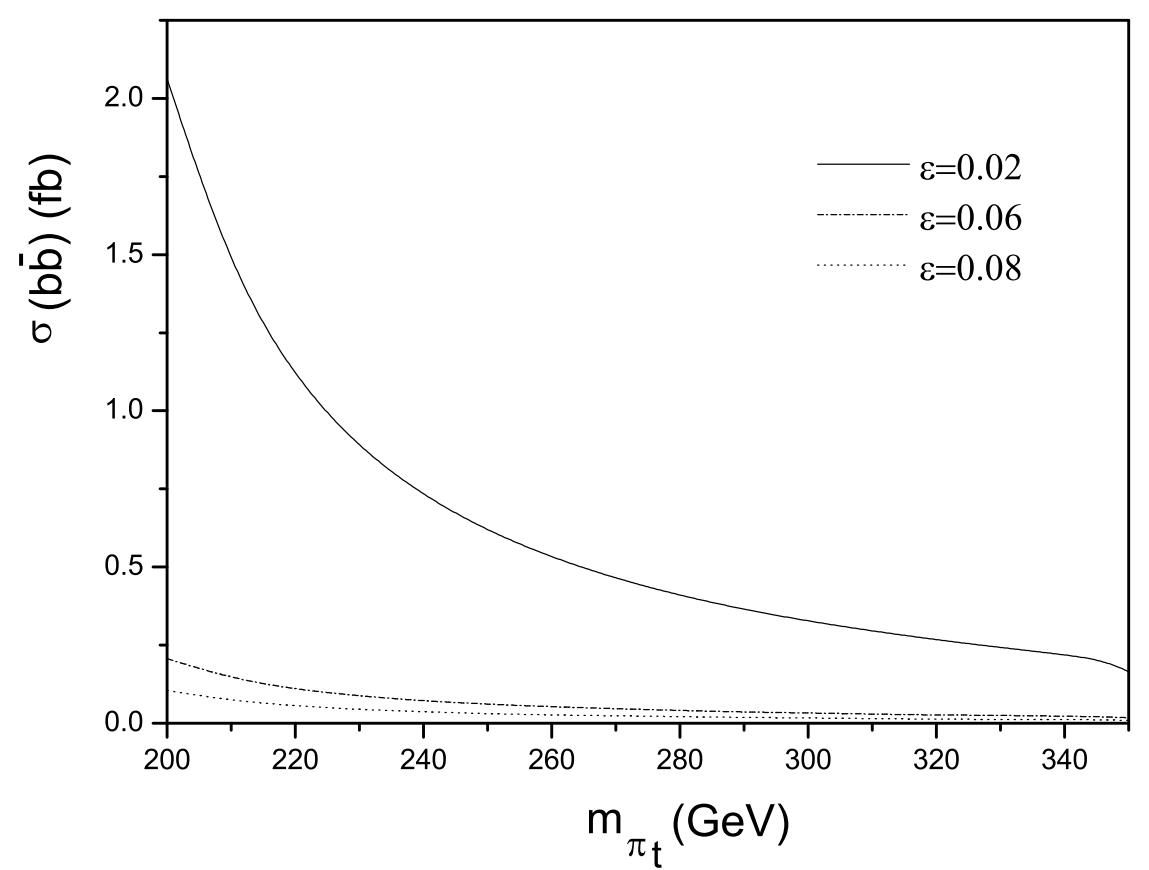

Fig.1 The resonance production cross section $\sigma(b \bar{b})$ versus the top-pion mass $m_{\pi_{t}}$ for three values of the parameter $\varepsilon$.

\section{The neutral top-pion $\pi_{t}^{0}$ and the process $\mu^{+} \mu^{-} \longrightarrow \bar{t} c$}

From section 2 we can see that the most dominant decay mode of the neutral top-pion $\pi_{t}^{0}$ is $\bar{t} c$ for $m_{\pi_{t}} \leq 350 \mathrm{GeV}$. Thus, compared to the process $\mu^{+} \mu^{-} \longrightarrow b \bar{b}, \pi_{t}^{0}$ can give significant contributions to the process $\mu^{+} \mu^{-} \longrightarrow \bar{t} c$ via the $\pi_{t}^{0}$ exchange in the s-channel. Furthermore, it is well known that there is no flavor changing neutral current (FCNC) at tree-level in the SM. The production cross sections of the FCNC process are very small at one-loop level due to the unitary of CKM matrix. The FCNC processes can be used to search for new physics. Any observation of the FC coupling deviated from that in the SM would unambiguously signal the presence of new physics. Thus, the process 
$\mu^{+} \mu^{-} \longrightarrow \pi_{t}^{0} \longrightarrow \bar{t} c$ will give a signal which should be easy to identify. The neutral top-pion $\pi_{t}^{0}$ may be easy detected via this process at the FMC.

In Fig. 2 we show the resonance production cross section $\sigma(\bar{t} c)$ as a function of the top-pion mass $m_{\pi_{t}}$ for $\sqrt{s}=m_{\pi_{t}}$ and three values of the parameter $\varepsilon$. We can see from Fig.2 that the $\sigma(\bar{t} c)$ decreases with $m_{\pi_{t}}$ and the parameter $\varepsilon$ increasing. For $m_{\pi_{t}} \geq 2 m_{t}$, the cross section $\sigma$ drops considerably since the $t \bar{t}$ channel opens up and the branching ratio $B_{r}\left(\pi_{t}^{0} \longrightarrow \bar{t} c\right)$ drops substantially. The value of the $\sigma(\bar{t} c)$ is larger than $3.7 \mathrm{fb}$ for $\varepsilon \leq 0.08$ and $200 G e V \leq m_{\pi_{t}} \leq 350 \mathrm{GeV}$. Thus, the FMC with $\sqrt{s}=200 \mathrm{GeV}-500 \mathrm{GeV}$ and a yearly integrated luminosity of $L=20 \mathrm{fb}^{-1}$ will generate tens and up to thousand $\bar{t} c$ events for $200 \mathrm{GeV} \leq m_{\pi_{t}} \leq 350 \mathrm{GeV}$ and $0.02 \leq \varepsilon \leq 0.08$. For example, it will generate $280 \bar{t} c$ events per year for $m_{\pi_{t}}=300 \mathrm{GeV}$ and $\varepsilon=0.06$. Thus, we can study the signals and observability of the neutral top-pion $\pi_{t}^{0}$ via the process $\mu^{+} \mu^{-} \longrightarrow \bar{t} c$ at the FMC.

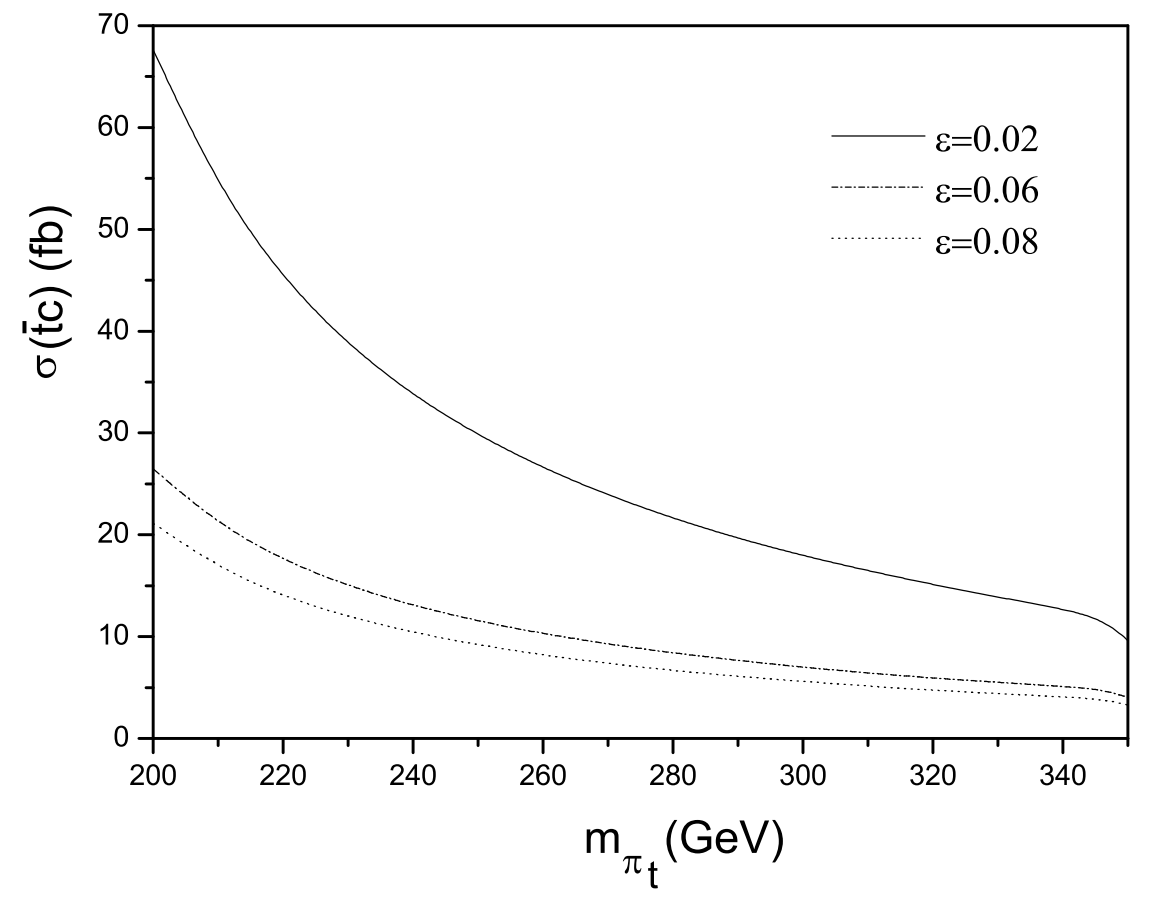

Fig.2 The resonance production cross section $\sigma(\bar{t} c)$ as a function of $m_{\pi_{t}}$ for three values of the parameter $\varepsilon$.

The parameter $\varepsilon$ of TC2 models represents the contributions of ETC interactions or other interactions to the mass of the top quark and is in the range of $0.02-0.1$ [8]. The 
current constraints on the free parameter $\varepsilon$ from low energy data (such as the $D-\bar{D}$ and $B-\bar{B}$ mixing and the $b \rightarrow s \gamma$ rate) are rather weak. To see the effects of the parameter $\varepsilon$ on the resonance production cross section $\sigma(\bar{t} c)$, we plot $\sigma(\bar{t} c)$ as a function of the parameter $\varepsilon$ for $\sqrt{s}=m_{\pi_{t}}=300 \mathrm{GeV}$ in Fig.3. We can see from Fig.3 that the cross section $\sigma(\bar{t} c)$ is larger than $5.6 \mathrm{fb}$ for $\varepsilon \leq 0.08$ and the number of yearly generated $\bar{t} c$ events is larger than 110 .

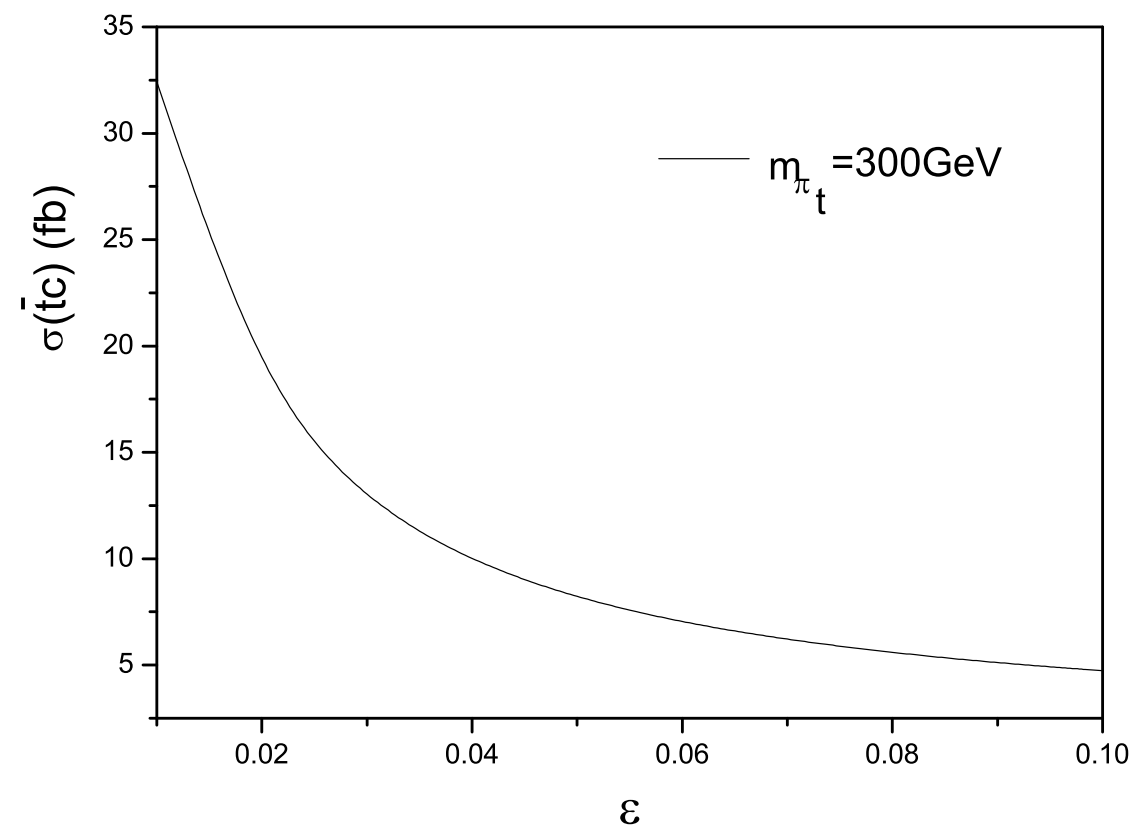

Fig.3 The resonance production cross section $\sigma(\bar{t} c)$ as a function of the parameter $\varepsilon$ for $\sqrt{s}=m_{\pi_{t}}=300$.

The possible observable final states of the FCNC process $\mu^{+} \mu^{-} \longrightarrow \bar{t} c$ are $\bar{b} c j_{1} j_{2}$, where $j_{1}$ and $j_{2}$ are light jets coming from $t \longrightarrow b W^{+}$followed by $W^{+} \longrightarrow u \bar{d}$ or $c \bar{s}$, and $\bar{b} c l^{+} \nu_{l}$, which come from $t \longrightarrow b W^{+} \longrightarrow b l^{+} \nu_{l}$ with $l=e, \mu$ or $\tau$. These two final states occur with branching of $2 / 3$ and $1 / 3$, respectively. The leading SM backgrounds of the FCNC process $\mu^{+} \mu^{-} \longrightarrow \bar{t} c$ mainly come from $W$ pair production and from $W$ bremsstrahlung in $\mu^{+} \mu^{-} \longrightarrow W+2$-jets. The techniques for suppressing this kind of backgrounds were discussed in Ref.[19].

We define the background-free observable cross section $\overline{\sigma(\bar{t} c)}$ as the effective cross 
section including b-tagging efficiency $\left(\varepsilon_{b}\right)$ and top quark reconstruction efficiency $\left(\varepsilon_{t}\right)$ : $\overline{\sigma(\bar{t} c)}=\varepsilon_{b} \varepsilon_{t} \sigma(\bar{t} c)$. In order to estimate the number of the observable events, we assume $\varepsilon_{b}=60 \%$ and $\varepsilon_{t}=80 \%$ as done in Ref.[19]. Then the yearly production observable $\bar{t} c$ events at the FMC can be easily estimated. The FMC with $\sqrt{s}=200 G e V-500 G e V$ and yearly integrated luminosity of $L=20 \mathrm{fb}^{-1}$ will generate 36 and up to 649 observable $\bar{t} c$ events for $200 \mathrm{GeV} \leq m_{\pi_{t}} \leq 350 \mathrm{GeV}$ and $0.02 \leq \varepsilon \leq 0.08$. Thus, it is easy to detect the neutral top-pion $\pi_{t}^{0}$ via the process $\mu^{+} \mu^{-} \longrightarrow \pi_{t}^{0} \longrightarrow \bar{t} c$. We can study the properties of the neutral top-pion $\pi_{t}^{0}$ via the process $\mu^{+} \mu^{-} \longrightarrow \bar{t} c$ at the FMC.

\section{Conclusions}

The top quark, with a mass of the order of the electroweak scale, is singled out to play a key role in the dynamics of EWSB and flavor symmetry breaking. There may be a common origin for EWSB and top quark mass generation. Much theoretical work has been carried out in connection to the top quark and EWSB. Various kinds of strong top dynamical models have been proposed, including TC2 models [8], flavor-universal TC2 models [20], top see-saw models [21], and top flavor see-saw models [22]. The common feature of such type of models is that topcolor interactions generate the main part of the top quark mass and also make small contributions to EWSB. EWSB is mainly generated by TC interactions or other interactions. Then, the presence of physical top-pions in the low energy spectrum is an inevitable feature of these models. Thus, studying the production and decay of these new particles at present or future high energy colliders is of special interest. It will be helpful to test the topcolor scenario and understand EWSB mechanism.

In this paper, we have studied the possible for detecting the neutral top-pion $\pi_{t}^{0}$ at the FMC via the processes $\mu^{+} \mu^{-} \longrightarrow \pi_{t}^{0} \longrightarrow b \bar{b}$ and $\mu^{+} \mu^{-} \longrightarrow \pi_{t}^{0} \longrightarrow \bar{t} c$. We find that the resonance produce cross section $\sigma(b \bar{b})$ is very small and in the range of $10^{-1} \mathrm{fb}-10^{-3} \mathrm{fb}$ in most of the parameter space. Furthermore, the backgrounds of the final state $b \bar{b}$ are very large, the value of $\frac{S}{\sqrt{B}}$ is smaller than 0.2 in all of the parameter space. Thus, it is very difficult to detect the neutral top-pion $\pi_{t}^{0}$ via this process. However, for the process 
$\mu^{+} \mu^{-} \longrightarrow \pi_{t}^{0} \longrightarrow \bar{t} c$, it is not this case. As long as the neutral top-pion mass $m_{\pi_{t}}$ is below the $t \bar{t}$ threshold, the $\pi_{t}^{0}$ can generate 36 and up to 649 observable $\bar{t} c$ events at the FMC with $\sqrt{s}=200 \mathrm{GeV}-500 \mathrm{GeV}$. Even we take the parameter $\varepsilon=0.08$ and $m_{\pi_{t}}=350 \mathrm{GeV}$, $\pi_{t}^{0}$ also can generate 36 observable $\bar{t} c$ events. Thus, the process $\mu^{+} \mu^{-} \longrightarrow \pi_{t}^{0} \longrightarrow \bar{t} c$ might give a signal which should be easy to identify. The properties of the neutral top-pion $\pi_{t}^{0}$ can be studied via the process $\mu^{+} \mu^{-} \longrightarrow \bar{t} c$ at a future muon collider.

\section{Acknowledgments}

This work was supported by the National Natural Science Foundation of China (90203005). 


\section{References}

[1] V. Barger, M. S. Berger, J. F. Gunion, T. Han, Phys. Rev. Lett. 75(1995)1462; Nucl. Phys. Proc. Suppl. 51A(1996)13; Phys. Rep. 286(1997)1; R. Casalbuoni et al., JHFP 9008(1999)011; V. Barger et al., hep-ph/0110340.

[2] C. Blöchinger et al., hep-ph/0202199; H. Frass et al., hep-ph/0303044

[3] E. Eichten, K. Lane and J. Womersley, Phys. Rev. Lett. 80(1998)5489; R. Casalbuoni, et al., hep-ph/9801243 K. Lane, hep-ph/9801385; C. T. Hill, hep-ph/9802216; B. A. Dobrescu, hep-ph/9802259.

[4] Chongxing Yue, Gongru Lu, Jiantao Li, J.Phys.G27(2001)913.

[5] D. Atwood, L. Reina and A. Soni, Phys. Rev. Lett. 75(1995)3800; M. Sher, Phys. Lett. B487(2000)151.

[6] E. H. Simmons, hep-ph/0110196 R. S. Chivukula and C. Holbling, hep-ph/0110214; T. L. Barklow, hep-ph/0112286.

[7] For recent reviews, see K. Lane, "Technicolor 2000", hep-ph/0007304, C. T. Hill and E. H. Simmons, hep-ph/0203079.

[8] C. T. Hill, Phys. Lett. B345(1995)483; K. Lane and E. Eichten, Phys. Lett. B352(1995)383; K. Lane, Phys. Lett. B433(1998)96; G. Cvetic, Rev. Mod. Phys. $71(1999) 513$.

[9] L. Randall and E. H. Simmons, Nucl. Phys. B380(1992)3; K. Lane, Phys. Lett. B357(1995)624; V. Lubicz and P. Santorelli, Nucl. Phys. B460(1996)3; E. Eichten, K. Lane, J. Womersley, Phys. Lett. B405(1997)305; R. Casalbuoni et al., Nucl. Phys. B555(1999)3; K. Lane, Phys. Rev. D60(1999)075007.

[10] Chongxing Yue, Yuping Kuang and Gongru Lu, J. Phys. G23(1997)163; Chongxing Yue, Qingjun Xu, Guoli Liu, Jiantao Li, Phys. Rev. D 63(2001)115002; Jun-jie Cao, Zhao-hua Xiong, Jin-Min Yang,Phys. Rev. D67(2003)071701. 
[11] K. Lane, K. R. Lynch, S. Mrenna and E. H. Simmons, Phys. Rev. D66(2002)015001;

A. Zerwekh, C. Dib and R. Rosenfeld, Phys. Lett. B549(2002)154.

[12] A. K. Leibovich and D. Rainwater, Phys. Rev. D65(2002)055012; Chongxing Yue, Yong Jia, Yangming Zhang, Hong Li, Phys. Rev. D65(2002)095010; Xuelei Wang, et al., Phys. Rev. D66(2002)075009; Xuelei Wang, et al., Phys. Rev. D66(2002)075013.

[13] G. Burdman, Phys. Rev. Lett.83(1999)2888.

[14] H.-J. He, C.-P. Yuan, Phys. Rev. Lett.83(1999)28; H.-J. He, S. Kanemura, C.-P. Yuan, Phys. Rev. Lett. 89(2002)101803.

[15] G. Burdman and D. Kominis, Phys. Lett. B403(1997)101.

[16] D. Kominis, Phys. Lett. B358(1995)312; G. Buchalla, G. Burdman, C. T. Hill, D. Kominis, Phys. Rev. D53(1996)5185.

[17] W. Loinaz and T. Takuchi, Phys. Rev. D60(1999)015005.

[18] Chongxing Yue, Yuping Kuang, Xuelei Wang, Weibin Li, Phys. Rev. D62(2000)055005.

[19] T. Han, J. L. Hewett, Phys. Rev. D60(1999)074015; S. Bar-Shalom, J. Wudka, Phys. Rev. D60(1999)094016.

[20] M. B. Popovic, E. H. Simmons, Phys. Rev. D58(1998)095007; G. Burdman and N. Evans, Phys. Rev. D59(1999)115005.

[21] B. A. Dobrescu, C. T. Hill, Phys. Rev. Lett.81(1998)2634; R. S. Chivukula, B. A. Dobrescu, H. Georgi, C.T. Hill, Phys. Rev. D59(1999)075003.

[22] H.-J. He, T. M. P. Tait, C.-P. Yuan, Phys. Rev. D62(2000)011702. 